\title{
Review
}

\section{Key stages in mammary gland development The cues that regulate ductal branching morphogenesis}

\author{
Mark D Sternlicht
}

Department of Anatomy, University of California, San Francisco, CA 94143-0452, USA

Corresponding author: Mark D Sternlicht, sternli@itsa.ucsf.edu

Published: 5 December 2005

This article is online at http://breast-cancer-research.com/content/8/1/201 (c) 2005 BioMed Central Ltd
Breast Cancer Research 2006, 8:201 (doi:10.1186/bcr1368)

and outgrowth of its earliest branches, its spatial organization via reiterative branching events and tissue remodeling, the formation of a continuous lumen, and tissue-specific differentiation of the entire network and its terminal structures. Indeed, many salient aspects of branching morphogenesis are well or partly understood [1-4], whereas many others are waiting to be solved. This article discusses our current sense and understanding of branching morphogenesis in the context of mammary gland development, with the realization that many of these notions also apply to other areas of development and disease.

The mammary gland, unlike other branched organs, undergoes most of its branching during adolescent rather than fetal development. In mice, mammary development begins shortly after mid-gestation, when bilateral epidermal ridges (or milk lines) form from forelimb to hindlimb, along which five pairs of disk-shaped placodes form at the site of each future nipple (this stage of mammary development is reviewed in the initial article in this series [5]). Each distinct placode then invaginates to form a bulb-shaped bud (the primary mammary rudiment or anlage) that penetrates the underlying mesenchyme and enters the cluster of preadipocytes that becomes the mammary fat pad. A limited number of branches then sprout from the invading anlage so that a rudimentary ductal tree occupying a small portion of the overall fat pad is present at birth. This rudimentary gland grows just enough to keep up with normal body growth until puberty, at which point robust hormone-dependent branching begins. At this stage, bulbous terminal end buds (TEBs) form at the tips of the ducts and penetrate farther into the fat pad as the ducts elongate (the biology of TEBs is reviewed more extensively in the second article in this series [6]). New primary ducts then form by bifurcation of the TEBs, and

$\mathrm{ADAM}=\mathrm{a}$ disintegrin and metalloproteinase; CSF-1 = colony-stimulating factor-1; ECM = extracellular matrix; EGFR = epidermal growth factor receptor; ER = estrogen receptor; FGF = fibroblast growth factor; FGFR = FGF receptor; GH = growth hormone; IGF-1 = insulin-like growth factor-1; IGF-1R = IGF-1 receptor; MMP = matrix metalloproteinase; PR = progesterone receptor; PTHrP = parathyroid hormone-related protein; $\mathrm{RANK}=$ receptor activator of nuclear factor $\mathrm{KB}$; TEB $=$ terminal end bud; TGF $=$ transforming growth factor; TIMP $=$ tissue inhibitor of metalloproteinases. 


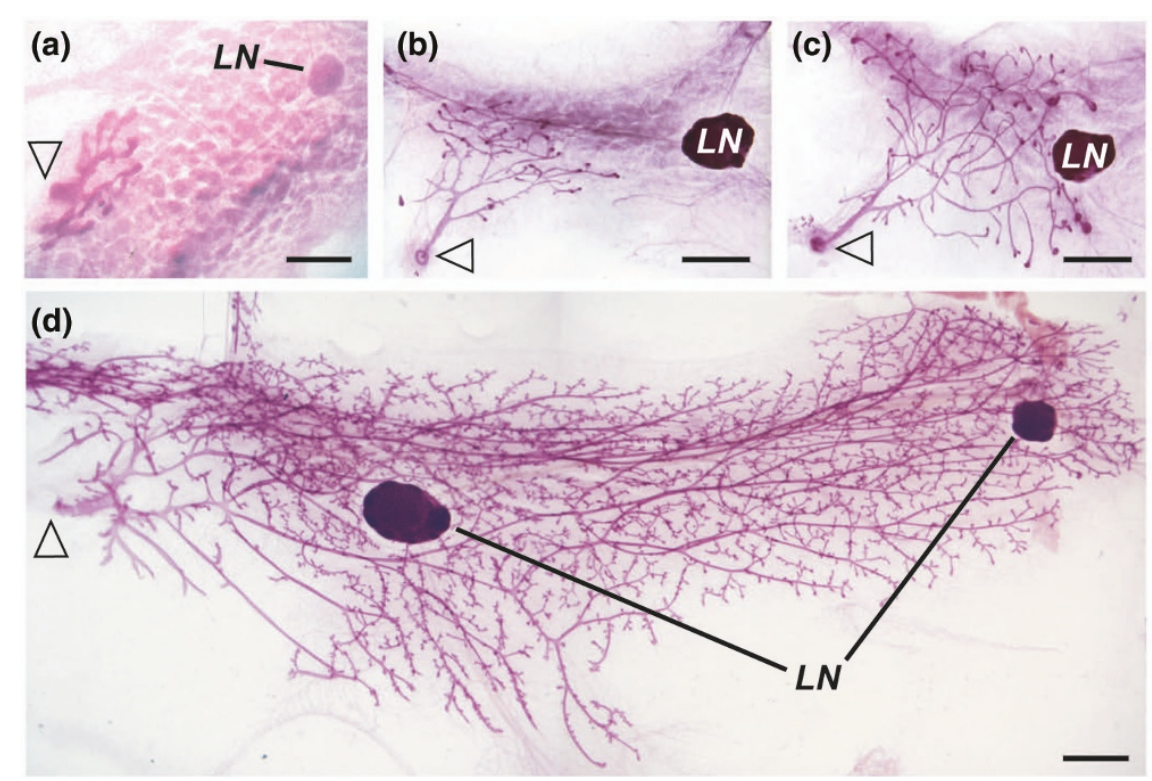

Nuclear-stained wholemounts illustrating ductal branching morphogenesis of the abdominal (no. 4) mammary gland. (a) Embryonic day 18.5; (b) age 3 weeks; (c) age 4.5 weeks; (d) age 11 weeks. Ductal penetration into the mammary fat pad can be judged with respect to the nipple and/or main lactiferous duct (arrowhead), central lymph node (LN), distal LN (as seen in (d)) and fat pad margins. Terminal end buds are readily apparent in the growing 4.5-week gland, and short tertiary branches are apparent in the mature 11-week gland. Scale bars, $0.5 \mathrm{~mm}(\mathrm{a})$ and $1 \mathrm{~mm}(\mathrm{~b}-\mathrm{d})$.

secondary side-branches sprout laterally from the trailing ducts until the entire fat pad of the young adult is filled by an extensive system of branched ducts (Figs 1 and 2). Thereafter, short tertiary side-branches form along the ducts in response to cycling ovarian hormones, further filling out the mature ductal tree. In addition, lobulo-alveolar structures develop like leaves at the ends of tertiary branches; however, the mechanisms that regulate their development and differentiation are distinct from those that govern ductal development (and will be reviewed in the next article in this series). Moreover, because some serial and limiting-dilution mammary transplants form pure ductal structures, whereas others form only alveolar outgrowths, distinct ductal and alveolar progenitor cells are probably scattered throughout the ductal tree.

Mammary development in humans is slightly different. Limited data from first-trimester human embryos also reveal the formation of a bilateral mammary ridge (or milk line) followed by the appearance of distinct placodes, the formation and ingrowth of mammary bulbs, and initial budding of the nascent mammary cone [7]. In addition, there is a conspicuous absence of hair pegs around the mammary anlagen reminiscent of lateral inhibition. As in mice, a rudimentary ductal tree then forms during the latter stages of embryonic development via progressive elongation, canalization and branching of the anlage. However, fetal exposure to maternal hormones results in limited secretory activity and the production of colostrum in the late-term fetus and newborn infant that is otherwise absent in rodents. Once these maternal influences subside, the infant breast undergoes menopausal-like involution, after which residual ductal structures persist in a relatively quiescent state until puberty. To this point, human breast development in males is indistinguishable from female breast development, whereas in mice, androgen-dependent condensation of the mesenchyme surrounding the neck of the mammary bud results in destruction of the male mammary rudiment on or near embryonic day 14. After puberty, the female human breast undergoes variable amounts of TEB formation, duct elongation, dichotomous and lateral branching, terminal duct lobular unit formation and stromal expansion, whereas the male breast remains quiescent but capable of further development under certain circumstances (such as gynecomastia).

Mammary branching may thus be separated into embryonic, adolescent and adult phases, each of which is differentially regulated. For instance, adolescent branching requires estrogen and estrogen receptor- $\alpha$ (ER- $\alpha$ ), adult tertiary sidebranching requires progesterone and its receptor (PR), and embryonic branching is hormone independent, because it occurs in mice lacking ER- $\alpha$, ER- $\beta$, PR or the receptors for growth hormone $(\mathrm{GH})$ and prolactin $[8,9]$. Branching is also coordinated by local cross-talk between the developing duct epithelium and nearby stromal cells. Indeed, tissue transplantation studies in which mammary epithelium and salivary mesenchyme [10] or skin epithelium and mammary mesenchyme [11] were recombined demonstrate that 

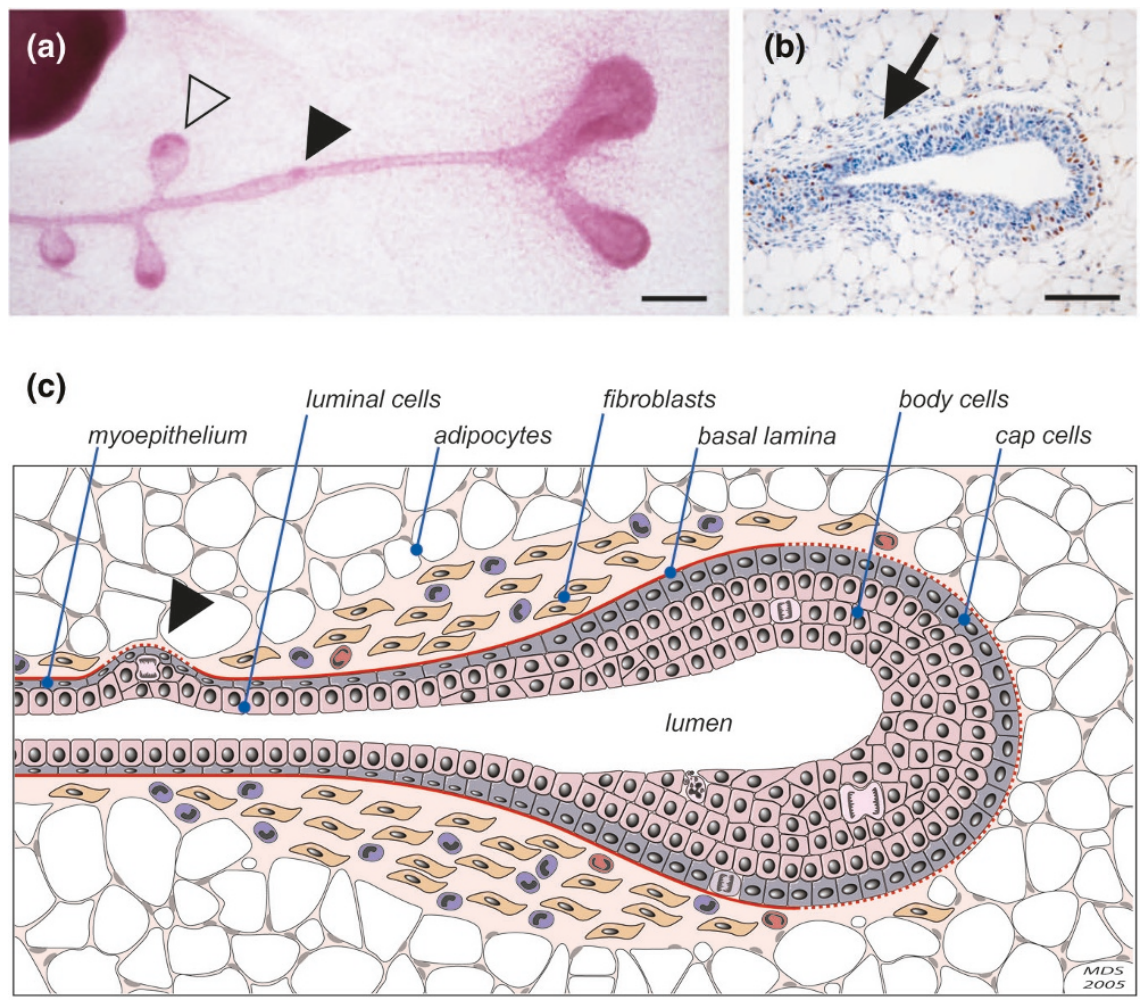

Terminal end bud (TEB) and duct morphology. (a) High-magnification carmine alum-stained wholemount of a primary duct that has recently passed the central lymph node (upper left corner). The bifurcating TEB is in the final stages of forming two new primary ducts with independent TEBs. Three newly formed lateral (secondary) side-branches are also present along the trailing duct (open arrowhead), as is an area of increased cellularity that may represent a nascent lateral bud (filled arrowhead). Increased stromal cellularity is also apparent about the bifurcating TEB. Scale bar, $200 \mu \mathrm{m}$. (b) Immunophotomicrograph of a TEB illustrating its considerable proliferative activity, as indicated by the large number of cells that have undergone DNA replication and have thus incorporated bromodeoxyuridine (brown diaminobenzidine-stained nuclei) during a 2-hour chase period. Rather than pulling themselves forward, TEBs seem to be pushed through the adipose-rich stroma by virtue of this high proliferative activity [6]. Hematoxylin counterstaining also reveals the stromal collar, rich in fibroblasts and collagen, that characteristically surrounds the TEB neck (arrow) and its conspicuous absence beyond the invading distal cap. Scale bar, $100 \mu \mathrm{m}$. (c) Schematic diagram depicting the salient architectural features of TEBs and their subtending ducts, including their fibroblast-rich stromal collar and high mitotic index. Though there is no evidence that normal ductal cells ever cross the basal lamina, thinning of the basement membrane (dotted lines) does seem to occur at the tips of invading ducts as a result of their partial enzymatic degradation and/or incomplete de novo synthesis. Stromal macrophages and eosinophils are also depicted.

mesenchymal cues control the branching pattern of the epithelium, regardless of epithelial origin. Likewise, similar studies show that stromal rather than epithelial or systemic factors dictate the different mammary side-branching patterns seen in different mouse strains [12]. A major difference between human and murine mammary glands is the dense, fibroblastic interlobular stroma and loose intralobular stroma of the human breast compared with the adipose-rich stroma seen in rodents. Nevertheless, xenotransplant studies support the notion that stromal influences also regulate human breast development. Indeed, the major mechanisms that regulate mammary morphogenesis are probably similar in all mammals. Thus this review outlines our basic understanding of the initial endocrine stimuli and local molecular interactions that regulate mammary branching in rodents as a model for mammary morphogenesis in general.

\section{Endocrine regulation of branching morphogenesis}

It has long been known that ovarian and pituitary hormones are essential for post-pubertal mammary morphogenesis and that estrogens can rescue mammary development in ovariectomized [13], but not hypophysectomized, animals [14]. However, estrogens can restore TEB and duct development in hypophysectomized, ovariectomized rats if $\mathrm{GH}$ or insulin-like growth factor-1 (IGF-1) is also provided, whereas pituitary prolactin will not suffice [14]. This suggests that $\mathrm{GH}$ is the critical pituitary hormone and that its effects are elicited through IGF-1. Indeed, adolescent ductal development is also impaired in mice lacking GH receptor [15], IGF-1 [14], ER- $\alpha$ [8], or the aromatase responsible for estrogen biosynthesis [16], but occurs normally in mice lacking ER- $\beta, P R$, or prolactin receptor [8], thus confirming the importance of $\mathrm{GH}$, 


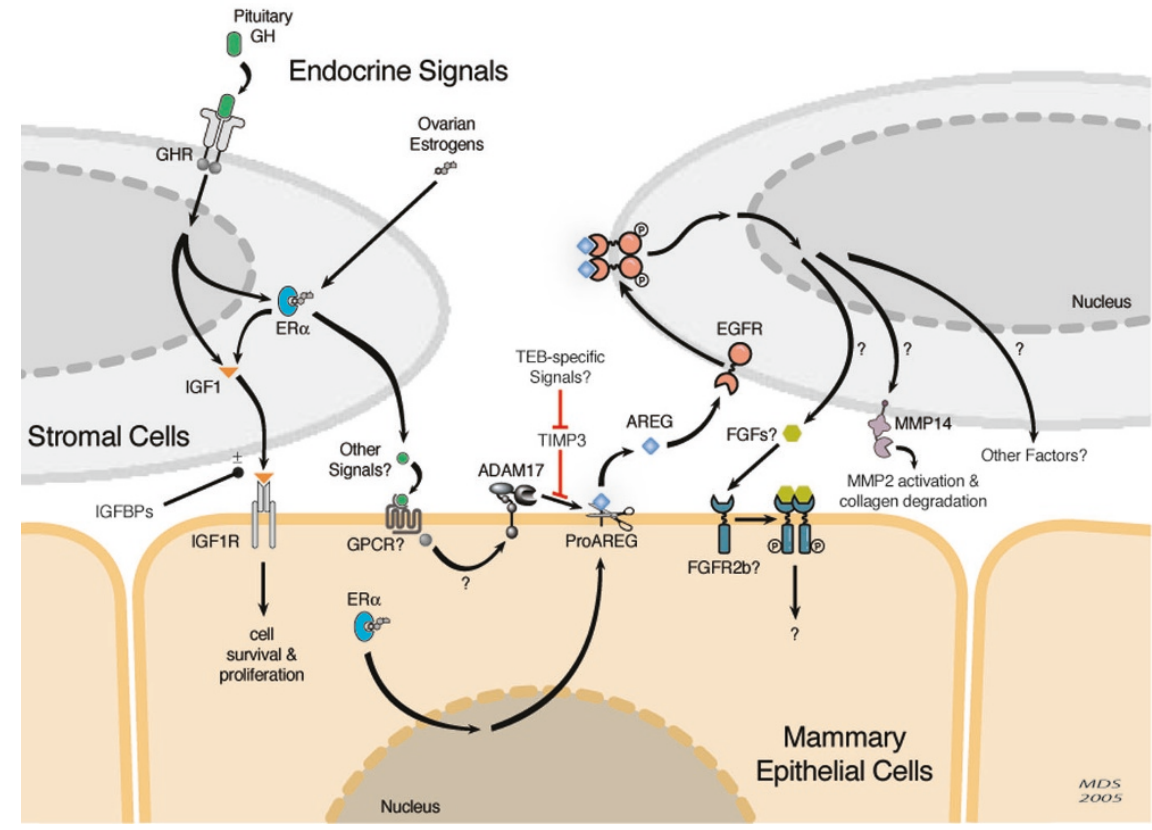

Provisional model depicting some of the key endocrine and paracrine signals that regulate mammary branching morphogenesis. ADAM, a disintegrin and metalloproteinase; AREG, amphiregulin; EGFR, epidermal growth factor receptor; ER, estrogen receptor; FGF, fibroblast growth factor; FGFR, FGF receptor; GH, growth hormone; GHR, GH receptor; IGF, insulin-like growth factor; IGF1R, IGF-1 receptor; IGFBPs, IGF-binding proteins; MMP, matrix metalloproteinase; TEB, terminal end bud; TIMP, tissue inhibitor of metalloproteinases.

IGF-1, estrogen and their respective receptors. Moreover, because IGF-1 rescues ductal development in $\mathrm{GH}$-deficient (hypophysectomized) animals, whereas excess $\mathrm{GH}$ and estrogen fail to rescue IGF-1-null glands, locally produced IGF-1 probably acts downstream of $\mathrm{GH}$ and/or estrogen [14]. Indeed, the importance of local versus systemic IGF-1 is supported by the observation that mammary branching is significantly diminished in mutant mice with globally reduced IGF-1 expression, but not in mice with a liver-specific deletion of IGF-1 and a resulting 75\% decrease in their circulating rather than mammary-specific IGF-1 levels [17]. IGF-1 receptor (IGF-1R)-deficient mammary transplants also show significantly reduced growth potential in surgically cleared (gland-free) wild-type fat pads [18], thus revealing the specific importance of epithelial rather than stromal IGF-1R, whereas similar experiments show that the $\mathrm{GH}$ receptor is only required in the stroma [15].

Embryonic tissue recombination studies also suggest that only stromal ER- $\alpha$ is required, whereas adult tissue transplants indicate that both epithelial and stromal ERs are required [19]. In addition, $\mathrm{GH}$ induces IGF-1 and ER expression in epithelium-free fat pads, the induction of IGF-1 is enhanced by estradiol, and only $\mathrm{GH}$-treated glands express stromal ER, further indicating that $\mathrm{GH}$ acts via the stroma [14]. These data therefore support the notion that pituitary $\mathrm{GH}$, which is already present before the pubertal surge in ovarian estrogens, acts via its receptor on mammary stromal cells to elicit the expression of IGF-1, that stromal IGF-1 then stimulates TEB formation and epithelial branching in a paracrine manner, and that ovarian estrogens act in concert with $\mathrm{GH}$ and IGF-1 to stimulate branching (Fig. 3).

Although estrogens induce PR expression, and progesterone can enhance IGF-1 activity to stimulate mammary ductal morphogenesis [20], the lack of ductal development in ER- $\alpha$-null mice is probably not due to diminished $P R$ function, because ablation of the gene that encodes the two PR isoforms PR-A and PR-B via alternative initiation has no effect on adolescent ductal development [21]. Nevertheless, the absence of both isoforms does block tertiary side-branching and lobuloalveolar development in adult and pregnant mice, and their selective ablation suggests that only PR-B is necessary and sufficient to elicit these effects. Tissue localization and recombination data also indicate that epithelial rather than stromal PRs stimulate lobuloalveolar development, whereas stromal PR may regulate tertiary branching [22,23]. Notably, Wnt4 is regulated by progesterone and is also required for tertiary side-branching [24]. Thus Wnt4 may act downstream of PR. Nevertheless, the consequences of its absence fade in late pregnancy, suggesting that other parallel pathways may also contribute. Indeed, receptor activator of NFKB (RANK) ligand may also participate, because it too is regulated by 
progesterone [25] and because pregnancy-associated lobuloalveolar development is impaired in knock-in mice harboring mutations in the NFKB activator I $\kappa B$ kinase- $\alpha$ and in mice lacking RANK or its ligand [26]. Otherwise, precisely how progesterone regulates mammary side-branching remains to be determined. Moreover, even though it is dispensable for normal primary and secondary branching, progesterone may still influence these processes in a nonessential manner, because it can enhance the morphogenetic effects of IGF-1 in the absence of estrogens, namely in ovariectomized IGF-1-null mice given IGF-1 plus progesterone [20].

\section{Local regulation of branching morphogenesis: the role of epidermal growth factor receptor (EGFR/ErbB1) and ErbB2}

EGFR is a receptor tyrosine kinase that elicits its effects on binding one of seven ligands and dimerizing with another EGFR monomer or one of three related ErbB receptors. Notably, EGFR ligands rescue ductal development in ovariectomized [27] and ER- $\alpha$-deficient mice [28], and exogenous estrogen elicits EGFR activation in ovariectomized mice [29], suggesting that EGFR promotes mammary branching downstream of ER- $\alpha$. Several EGFR ligands promote mammary development if given exogenously; however, amphiregulin is the only one that is upregulated at puberty and the only one that is required, because ductal outgrowth is impaired in amphiregulin-deficient mice but not in mice lacking EGF, transforming growth factor- $\alpha$ (TGF- $\alpha$ ), heparin-binding EGF-like growth factor, or betacellulin $[30,31]$. EGFR is also required, but only in the stroma, whereas its key ligand amphiregulin is exclusively expressed and required in the epithelium [27,29-31]. Thus amphiregulin, which is expressed as a transmembrane precursor, must be proteolytically shed from the epithelial cell surface to activate EGFR on nearby stromal cells. Notably, the transmembrane metalloproteinase ADAM (a disintegrin and metalloproteinase) 17 (TNF- $\alpha$-converting enzyme; TACE) can release amphiregulin and other EGFR ligands in culture. Moreover, ADAM17-null mice resemble EGFR-null mice in many respects, including the failure of their mammary glands to develop when transplanted to viable hosts [31]. Furthermore, as one would expect, ADAM17 is only required in the same location as its apparent substrate, local amphiregulin administration rescues ADAM17-null transplants, and EGFR phosphorylation occurs only when ADAM17 and amphiregulin are expressed on mammary epithelial cells and EGFR is present in the stroma. Thus ADAM17 has an essential role in the epithelial-stromal cross-talk that regulates mammary development by liberating an essential ligand (amphiregulin) that is expressed only on epithelial cells so it can activate its receptor (EGFR) on stromal cells.

The persistent failure of ADAM17-deficient glands to catch up over time despite the presence of other related enzymes also means that ADAM17 is the only physiologic sheddase for amphiregulin and/or that it is independently regulated. Interestingly, the only endogenous inhibitor of ADAM17, tissue inhibitor of metalloproteinases 3 (TIMP-3), is specifically downregulated in TEBs (but not trailing ducts), whereas TIMP-1 is specifically upregulated [31]. This would tend to enhance ADAM17-dependent processing of amphiregulin and EGFR activation in an apt location, while limiting the activity of other TIMP-1-inhibitable enzymes. In addition, estrogen is a potent inducer of amphiregulin, and Gprotein-coupled receptors can stimulate ADAM17-mediated EGFR transactivation in culture. However, the specific cues that elicit ADAM17 activity during mammary development are not yet known.

Nor is it clear what lies downstream of EGFR. Because TIMP1 inhibits mammary branching in culture and in vivo [32], but does not inhibit ADAM17, at least one other metalloproteinase must be involved, and because metalloproteinase inhibitors block branching in culture in response to EGFR agonists [32], they are probably inhibiting enzymes that act downstream of EGFR. By contrast, the absence of ADAM17 does not preclude branching in response to EGFR agonists [31] because it acts upstream. Notably, EGFR activation stimulates the expression of matrix metalloproteinase (MMP)-2 (gelatinase-A) and the MMP-2 activator MMP-14 (MT1-MMP), and the EGFR-dependent induction of MMP-14 and subsequent activation of MMP-2 have a key role in lung branching morphogenesis [33]. Moreover, MMP-2 regulates mammary ductal elongation in vivo; MMP-3 (stromelysin-1), which has not been linked to EGFR signaling, regulates sidebranching; and MMP-14, which is induced in the presumably activated stromal cells surrounding TEBs, promotes ductal development by activating MMP-2 and collaborating with it to degrade type I collagen [31,32].

The possibility that fibroblast growth factors (FGFs) regulate branching downstream of EGFR (or in a parallel pathway) is supported by the observation that FGFs 2 and 7 support the growth and branching of cultured EGFR-null mammary organoids [31], whereas EGFR agonists and FGFs fail to support the growth of organoids lacking FGF receptor 2 (FGFR2) [34]. Notably, FGFR2b is expressed on mammary epithelial cells and is required for forming embryonic mammary placodes, as is stromal FGF10 [5]. Moreover, conditional ablation of FGFR2 causes a severe delay in adolescent ductal development, and an analysis of genetic mosaicism reveals that epithelia without FGFR2 are eliminated from the ducts that do develop [34]. However, no mammary phenotype has been described in FGF7-deficient mice, possibly as a result of compensatory mechanisms, and it remains unclear whether other FGF receptors or receptor isoforms are involved. Nevertheless, stromal FGFs and their epithelial receptors have key roles in branching of the Drosophila tracheal system and in mammalian lung, salivary gland and kidney branching, suggesting that similar mechanisms may also influence mammary branching [1]. 
Evidence also indicates that the transmembrane tyrosine kinase and potential EGFR partner ErbB2 influences ductal morphogenesis. Transplantation of genetically rescued ErbB2-null mammary glands to cleared wild-type fat pads [35] and selective ablation of ErbB2 in mammary epithelial cells [36] causes TEB defects and delays ductal penetration, indicating that epithelial ErbB2 is required. However, because ErbB2 has no known ligand, it requires a coreceptor; yet ErbB4 and epithelial EGFR are expendable and ErbB3 is weakly expressed during ductal development $[29,31,37]$. Thus it remains unclear how ErbB2 regulates ductal development or whether epithelial EGFR-ErbB2 heterodimers participate in ways that were not specifically addressed by examining recombined EGFR-null transplants, such as whether they influence the rate of ductal development.

\section{Factors that regulate embryonic mammary branching}

Some pathways may affect only the formation of the embryonic ductal tree, others may affect only its postnatal maturation, and still others may affect both prenatal and postnatal development. However, the postnatal role of those pathways that are essential for initial mammary development may be difficult to ascertain if, in their absence, the embryonic mammary rudiment fails to form in the first place, thus necessitating the use of conditional approaches. For instance, mice lacking the estrogen-regulated homeobox transcription factor MSX2 form mammary buds that fail to undergo embryonic branching, thus concealing any role that MSX2 might have in adolescent branching [5]. Similarly, instructive paracrine signaling between parathyroid hormonerelated protein $(\mathrm{PTHrP})$ from the embryonic mammary bud epithelium and its receptor PTHR1 on adjacent mesenchymal cells is required for the formation of mammary-specific mesenchyme, which is, in turn, required in forming a rudimentary ductal tree [5]. Thus it is also unclear whether PTHrP participates in subsequent branching steps, although its overexpression during adolescent development slows ductal elongation by increasing apoptosis in TEBs, and its overexpression during embryogenesis somehow diminishes ductal branching later in life [38]. Embryonic mammary glands are also arrested at the bud stage in mice lacking the LEF1 transcription factor that lies downstream of the canonical (namely the $\beta$-catenin-dependent) Wnt signaling cascade, whereas buds fail to form at all in transgenic mice that express the diffusible Wnt inhibitor Dickkopf-1 under the control of an epidermal (keratin14) gene promoter [5]. Nevertheless, Wnts participate in the branching of other tissues, their transgenic overexpression affects mammary branching, and mammary-targeted expression of an inhibitory form of a Wnt receptor that blocks both canonical and noncanonical Wnt signaling delays adolescent ductal development (AMC Brown, personal communication), suggesting that at least one Wnt signaling pathway is involved in mammary ductal development.
The broad systemic or lethal effects of ablating a particular molecule may also obscure its local role in embryonic and adolescent branching, although the latter can often be addressed through transplantation. For example, a recent study found that development of the embryonic mammary tree is significantly impaired in both ADAM17-null and EGFRnull mice, yet a previous examination of EGFR-null newborns revealed no such impairment [31]. It is therefore unclear whether the observed delay in embryonic branching reflects the generalized runting that results from EGFR or ADAM17 ablation, strain-specific genetic effects, the lack of local EGFR signaling, or a combination thereof.

\section{Ductal morphology versus branching}

Although it is reasonable to predict that mechanisms that affect TEB and duct morphology should also affect branching, this may not always be true. Netrin-1, which acts as a diffusible attractant and repellent during neuronal guidance, is also secreted by the body cells of mammary TEBs, whereas its receptor neogenin is expressed on adjacent cap cells [39]. The absence of either netrin-1 or neogenin causes dissociation of the cap and body cell compartments and inappropriate migration of cap cells into the preluminal compartment. Moreover, neogenin mediates netrin-dependent cell clustering, further indicating that netrin-neogenin interactions stabilize the cap cell layer and mediate its adhesion to the preluminal body cells. Nevertheless, the absence of netrin-1 or neogenin has no effect on overall branching, suggesting that ductal patterning and TEB morphology do not depend on one another in this setting (L Hinck, personal communication).

Hedgehog signaling, which is elicited by the binding of Indian, Sonic or Desert hedgehog to cell surface Patched receptors, can affect several signaling pathways that are thought to regulate mammary branching, including the FGF, Wnt, Notch, TGF- $\beta$, and PTHrP pathways [40]. Mammary transplants lacking Indian or Sonic hedgehog branch normally in cleared wild-type fat pads, indicating that neither ligand alone is essential in the epithelium; however, conditional haploinsufficiency of Patched-1 causes defects in duct and TEB histology that disappear after transplantation to wild-type fat pads, suggesting that only stromal Patched-1 is required. Nevertheless, the overall branching pattern is unaffected. Moreover, transplants lacking the transcription factor Gli2 that lies downstream of Patched also display normal ductal branching despite their abnormal intra-ductal morphology, again suggesting that ductal patterning and morphology may not be entirely interdependent.

Conversely, some regulators, such as ErbB2, do seem to influence both ductal morphology and branching [35]. For example, the cell surface morphogen epimorphin is required for growth-factor-induced branching of organotypic mammary cultures and affects luminal diameter when provided in an apolar manner in culture or as a mammary-targeted transgene 
in vivo [41]. Its metalloproteinase-dependent release from stromal fibroblasts seems to be required for epimorphin to affect epithelial cells, although myoepithelial epimorphin could potentially act in a juxtacrine manner without being shed. As a downstream consequence, epimorphin stimulates the expression of MMPs 2 and 3, which are also required for proper branching morphogenesis. In addition, epimorphin stimulates expression of the transcription factor C/EBP $\beta$, which is also essential for mammary morphogenesis, and increases the relative expression of the shorter of two $\mathrm{C} / \mathrm{EBP} \beta$ isoforms. Indeed, experimental manipulation of the relative expression of these isoforms in the absence of epimorphin signaling has the same morphogenic effect in culture as epimorphin itself, suggesting that C/EBP $\beta$ acts downstream of epimorphin.

\section{The role of macrophages and eosinophils}

Among the stromal cells that influence mammary development, macrophages and eosinophils have a particularly important role in ductal elongation and branching [42]. Macrophage recruitment to the stroma surrounding TEBs, TEB formation and adolescent ductal outgrowth are severely impaired in myelosuppressed (gamma-irradiated) mice and mice lacking macrophage-colony-stimulating factor-1 (CSF-1) or its receptor, but are rescued by bone marrow transplantation in irradiated mice and by exogenous CSF-1 or a mammarytargeted CSF-1 transgene in CSF-1-null mice. Thus the essential effects of CSF-1 on macrophage behavior are local rather than systemic. Indeed, in the developing gland, ductal cells produce CSF-1, whereas its receptor is expressed exclusively on macrophages. Nevertheless, it remains unclear whether macrophages influence ductal development via their trophic, angiogenic, phagocytic, or matrix remodeling activities.

As regards eosinophils, their recruitment to the TEB stroma coincides with local upregulation of their chemoattractant eotaxin, which is recognized by the $\mathrm{CC}$ chemokine receptor CCR3, and such recruitment is severely diminished in eotaxin-deficient mice (as is ductal branching, but not elongation) [42]. In contrast, absence of the eosinophil chemotactic factor interleukin-5 causes a deficiency in circulating eosinophils but has no effect on the number of mammary eosinophils or on mammary development itself, further supporting the importance of eotaxin as a local chemoattractant for eosinophils during mammary development. Although the means by which eosinophils promote ductal branching is not entirely clear, mammary eosinophils secrete the chemokine C10, which seems to promote macrophage recruitment further. Thus eosinophils and macrophages may collaborate to bring about proper ductal morphogenesis.

\section{Negative regulators of branching morphogenesis}

Although controls against precocious, accelerated, or excess branching undoubtedly exist, a full understanding of their individual importance is difficult to obtain in the face of redundant or unrelated mechanisms. For example, the endogenous MMP inhibitor TIMP-1 defies ductal development in a gain-of-function setting, yet its absence has little or no effect on branching in a loss-of-function setting [32]. Thus it is unclear whether TIMP-1 is truly involved or compensated for by other TIMPs, because other restraints as prevalent as limits on the rate of cell proliferation undoubtedly continue to exert their own rate-limiting effects. Nevertheless, each of the branching agonists outlined in this review has its own negative regulators (Table 1 ), some of which might accelerate branching morphogenesis if lost. Indeed, some examples of accelerated ductal development after gene inactivation have also been seen. For instance, increased ductal invasion occurs when the receptor tyrosine kinase signaling antagonist Sprouty2 is inactivated in mammary epithelium, suggesting its possible importance in controlling FGFR2 signaling [34].

Considerable evidence also indicates that TGF- $\beta 1$ acts as a key negative regulator of mammary branching by limiting epithelial proliferation and stimulating extracellular matrix (ECM) production $[43,44]$. Notably, TGF- $\beta 1$ is regulated by ovarian hormones. Moreover, mammary-targeted expression of activated TGF- $\beta 1$ causes the formation of a hypomorphic ductal tree; slow-release TGF- $\beta 1$ implants inhibit epithelial proliferation, TEB formation and local ductal elongation; and most notably, heterozygous TGF- $\beta 1$-deficient mice, which have less than $10 \%$ of normal TGF- $\beta 1$ levels, exhibit two to four times more proliferation than normal (15-fold more in response to exogenous ovarian hormones) and significantly accelerated but morphologically normal ductal development. In addition, TGF- $\beta 1$ heterozygous glands exhibit accelerated outgrowth in wild-type fat pads, indicating that the growth inhibitory effects of TGF- $\beta 1$ are epithelial in origin. Nevertheless, these effects seem to be performed through both autocrine feedback mechanisms and paracrine interactions that may involve stromal type II TGF- $\beta$ receptors and reciprocal stromal responses. Thus TGF- $\beta 1$ may aid in the maintenance of proper ductal spacing by enabling neighboring ducts to avoid one another. Moreover, TGF- $\beta$ downregulates MMP-3 expression, which would tend to defy secondary sidebranching without affecting ductal elongation, whereas it upregulates MMP-2 expression, which would foster ductal elongation and thus increase the distance between secondary branch-points $[32,45]$.

\section{ECM-mediated regulation of branching morphogenesis}

Substantial evidence also indicates that ECM, ECM receptors and ECM-degrading enzymes are important regulators of branching morphogenesis [46]. Numerous culture-based studies show that, in addition to providing a structural foundation for cells, ECM components convey contextual information through cellular adhesion molecules, such as integrins, that transmit external ECM-derived signals 
Table 1

\begin{tabular}{|c|c|c|c|c|}
\hline Regulator & Agonists & Antagonists & Targets & Effects \\
\hline $\mathrm{GH}-\mathrm{R}(\mathrm{st})$ & $\mathrm{GH}$ (pit) & & IGF-1 (st) & General \\
\hline IGF-1R (ep) & IGF-1 (st) & IGFBPs (st) & Unknown & General \\
\hline ER- $\alpha$ (st/ep) & E1/E2 (ov) & & Amphiregulin et al. & General \\
\hline PR-B (st?) & $\mathrm{ER}, \mathrm{P}$ (ov) & & Wnt4, RANKL & Tertiary branching \\
\hline EGFR (st) & Amphiregulin (ep) & Sprouty? & MMPs, FGFs et al. & General \\
\hline Amphiregulin (ep) & ER, ADAM17 (ep) & HSPGs & EGFR (st) & General \\
\hline ADAM17 (ep) & PPCs et al. & TIMP3 (ep) & Amphiregulin (ep) & General \\
\hline MMP-14 (st) & EGFR, PPCs et al. & TIMPs 2-4 & MMP-2, Coll I & General \\
\hline MMP-2 (st?) & TGF- $\beta$, ER, MMP-14 & TIMPs $1-4$ & Coll I et al.? & Elongation \\
\hline MMP-3 (st) & Epimorphin et al. & TGF- $\beta$, TIMPs & Unknown & Secondary branching \\
\hline FGFR2 (ep) & FGFs (st) & Sprouty2 (ep) & Unknown & TEBs \\
\hline ErbB2 (ep) & Unknown & Unknown & Unknown & TEBs \\
\hline Epimorphin (st/me) & Metalloenzymes & Unknown & C/EPB $\beta$, MMPs & Branching \\
\hline TGF- $\beta 1$ (ep) & ER, MMPs, plasmin & LAP & TGF- $\beta$ RI/II, MMPs & Spacing \\
\hline CSF-1R (m $\phi)$ & CSF-1 (ep) & & $\mathrm{M} \phi$ recruitment & TEBs \\
\hline CCR3 (eos) & Eotaxin & & Eos recruitment & Branching \\
\hline Integrin $\alpha_{2} \beta_{1}$ (ep) & Laminins, collagens & & & TEBs, ducts \\
\hline DDR1 (ep) & Collagens & & & TEBs, ducts \\
\hline
\end{tabular}

ADAM, a disintegrin and metalloproteinase; CCR, CC chemokine receptor; Coll, collagen; CSF, colony-stimulating factor; DDR, discoidin domain receptor; EGFR, epidermal growth factor receptor; eos, eosinophil; ep, epithelial; E1/E2, estrone/estradiol; ER, estrogen receptor; FGF, fibroblast growth factor; FGFR, FGF receptor; GH, growth hormone; GH-R, growth hormone receptor; HSPG, heparan sulfate proteoglycan; IGF-1, insulinlike growth factor-1; IGF-1R, IGF-1 receptor; IGFBPs, IGF-binding proteins; LAP, latency-associated peptide; me, myoepithelial; MMP, matrix metalloproteinase; $m \phi$, macrophage; ov, ovarian; pit, pituitary; PPC, proprotein convertase; P, progesterone; PR, progesterone receptor; RANKL, receptor activator of nuclear factor KB (RANK) ligand; st, stromal; TEB, terminal end bud; TGF, transforming growth factor; TIMP, tissue inhibitor of metalloproteinases. Required sources are provided in parentheses.

to the cell interior. Indeed, the three-dimensional ECM environment has been shown to affect virtually all aspects of cell behavior, including cell shape, proliferation, survival, migration, differentiation, polarity, organization, and branching. Notably, $\alpha_{2}$ integrin-deficient mice, which are unable to form integrin $\alpha_{2} \beta_{1}$ collagen/laminin receptors, show significantly diminished ductal branching, but have otherwise normal ducts and are fully able to nurse their pups [47]. Furthermore, function-perturbing antibodies against the $\beta_{1}$ integrin subunit cause TEB regression and diminished ductal elongation, as do antibodies against the $\gamma_{1}$ chain found in most laminins, which in turn are the main basement membrane ligands for $\beta_{1}$ integrins [48]. These, however, are relatively mild effects compared with the more severe branching defects that occur when $\alpha_{2} \beta_{1}$ integrin function is blocked or absent in culture. Similarly, $\alpha_{3}, \alpha_{6}$, and $\beta_{4}$ integrins can affect branching in culture, but are not required in vivo [49]. Thus the combined effects of multiple ECM receptors in vivo may mitigate the effects of disrupting a single interaction. Indeed, evidence suggests that other, non-integrin receptors may also be involved. For example, mice lacking the discoidin domain receptor tyrosine kinase DDR1 that signals in response to binding triple-helical fibrillar collagens exhibit delayed, abnormal ductal development [50]. Indeed, the importance of fibrillar collagens as signaling molecules rather than as mere scaffolds or barriers may help to explain the diminished ductal development seen in mice that lack collagenolytic MMPs 2 or 14 or that express cleavageresistant as well as hyper-cleavable type I collagen (M Egeblad, MDS, BS Wiseman and Z Werb, unpublished results). Likewise, the transgenic overexpression of $\beta-1,4$ galactosyltransferase, a receptor for laminin and other glycosylated proteins, causes abnormal and diminished ductal development [51], and the laminin receptor dystroglycan may also participate, because it affects mammary epithelial cells in culture and affects branching morphogenesis in other tissues [52].

In addition to their direct effects, various ECM components bind and sequester other signaling molecules that affect 
branching, such as amphiregulin, FGFs, Wnts, TGF- $\beta$, and IGF-binding proteins 1 to 6 . Thus enzyme-mediated ECM remodeling can remove physical barriers and existing ECM signals, reveal hidden structural information, and release otherwise sequestered signaling molecules. Indeed, ECMdegrading MMPs seem to have a path-clearing role in branching morphogenesis as well as an indirect cell signaling role that may reflect their ability to alter extant ECM signals, generate bioactive ECM fragments (for example cryptic integrin-binding sites on fibrillar collagen and a laminin-5 fragment that elicits epithelial cell motility), cleave cell-cell adhesion proteins (for example E-cadherin), remove cell surface receptors (for example FGFR1), release ECM-bound growth factors, inactivate IGF-binding proteins, activate latent TGF- $\beta 1$, and recruit other cell types to the surrounding stroma [32,45]. In addition, the spatial deposition of new matrix barriers is likely to influence ductal branch-point selection. For example, localized fibronectin deposition is essential for salivary gland cleft formation and is associated with a switch from E-cadherin-mediated cell-cell adhesion to $\alpha_{5} \beta_{1}$ integrin-mediated cell-matrix adhesion [53]. Thus similar mechanisms may also influence mammary branching.

Another fundamental aspect of ductal morphogenesis that depends, in part, on the three-dimensional matrix microenvironment is lumen formation; that is, the assembly of hollow polarized cysts (alveoli) and tubes (ducts). This process seems to be driven by an intrinsic program whereby adherent epithelia seek to establish basal, lateral, and free apical surfaces via cell-cell, cell-matrix and associated cytoskeletal interactions [4]. In addition, canalization may involve anti-adhesive mechanisms that promote the separation of apposed membranes and the apoptotic or autophagic removal of cells from the luminal space. In conjunction with these hard-wired mechanisms for forming polarized luminal structures, organotypic culture data suggest that new mammary ducts and alveoli develop by a continuous 'rubber-sheet deformation' mechanism in which free, lateral, and basal cell surfaces are maintained as the adherent epithelia bulge outward to form new hollow evaginations [4]. In addition, the tunneling process that follows invading TEBs seems to involve the clearance of centrally located body cells by apoptosis. Thus mammary tubulogenesis seems to involve both budding and cavitation rather than any of the other mechanisms by which tubular tissues can develop (as reviewed elsewhere $[3,4]$ ).

Notably, mammary ducts are bilayered tubes composed of inner luminal epithelial cells surrounded by myoepithelial cells, which are in turn surrounded by an extracellular basement membrane. In addition to fostering oxytocin-induced milk ejection by virtue of their contractile activity, myoepithelial cells are the cells that actually contact the basement membrane directly and are required for the production of many of its components, including laminins. Thus they are ideally situated to transmit structural morphogenetic information from the basement membrane to the luminal epithelia. Indeed, isolated luminal epithelial cells (which do not form their own basement membranes) fail to form properly polarized hollow spheres when cultured in type I collagen gels and instead form solid lumen-less structures with reverse polarity unless myoepithelial cells are also added, in which case they do form aptly polarized, hollow, bilayered acinar-like structures [54]. Moreover, laminin-1 or minor amounts of reconstituted basement membrane (but not laminins 5 or $10 / 11$ ) are also able to rescue the polarity of cultured luminal cells in the absence of myoepithelial cells. Conversely, alveolar morphogenesis (that is, the proper positioning of luminal and myoepithelial cells) is blocked by peptides that interfere with their desmosomal cell-cell interactions [55]. Thus myoepithelial cells and the basement membrane components that they produce (particularly laminin-1) seem to provide a key link in establishing proper ductal polarity and lumen formation, as does their cell-matrix adhesion and their desmosomal adhesion to neighboring luminal cells.

\section{Perspectives}

Several organs undergo branching morphogenesis and there are clear differences in how branching proceeds in different tissues and species. Nevertheless, many of the major mechanisms that regulate branching in one tissue are likely to be evolutionarily conserved among all branched organs and organisms. Indeed, many mechanistic similarities have been seen between different branched tissues in species as dissimilar as flies and mice [1]. However, there must also be unique mechanisms that elicit tissue-specific and speciesspecific differences. The challenge, of course, is to decipher these ubiquitous and unique mechanisms. In addition, the various types of mammary branching that occur (primary, secondary and tertiary) seem to use distinct mechanisms both at the level of signaling (that is, the cues that initiate and orchestrate their formation) and at the level of their physical formation (for example, the bifurcation of TEBs to form primary ducts differs from the eruption of secondary side-branches). However, despite considerable headway, our understanding of the complex cascade of signals that pass back and forth between neighboring cells of developing tissues, their systemic regulation, and the role of the matrix microenvironment is still largely incomplete, leaving the field ripe for further progress. Moreover, the same general processes, from proliferation to invasion, that take place during normal mammary development also occur in malignant disease, and most of the developmental pathways that influence branching have been associated, to some degree or other, with the development or progression of cancer. Thus a better understanding of the mechanisms that regulate mammary branching morphogenesis should provide critical new insight into other normal and pathologic processes.

\section{Competing interests}

The author(s) declare that they have no competing interests. 


\section{This article is part of a review series on Key stages in mammary gland development, edited by Charles Streuli.}
Other articles in the series can be found online at http://breast-cancer-research.com/articles/ review-series.asp?series=bcr_keystages

\section{Acknowledgements}

The author is especially grateful for the guidance and support of $\mathrm{Dr}$ Zena Werb. This work was supported by grants from the National Cancer Institute (CA57621 and CA58207) and a grant jointly funded by the National Institute of Environmental Health Sciences and the National Cancer Institute (ES012801).

\section{References}

1. Affolter M, Bellusci S, Itoh N, Shilo B, Thiery JP, Werb Z: Tube or not tube: remodeling epithelial tissues by branching morphogenesis. Dev Cell 2003, 4:11-18.

2. Davies JA: Watching tubules glow and branch. Curr Opin Genet Dev 2005, 15:364-370.

3. Lubarsky B, Krasnow MA: Tube morphogenesis: making and shaping biological tubes. Cell 2003, 112:19-28.

4. O'Brien LE, Zegers MMP, Mostov KE: Building epithelial architecture: insights from three-dimensional culture models. Nat Rev Mol Cell Biol 2002, 3:531-537.

5. Hens JR, Wysolmerski JJ: Key stages of mammary gland development: molecular mechanisms involved in the formation of the embryonic mammary gland. Breast Cancer Res 2005, 7:220-224.

6. Hinck L, Silberstein GB: Key stages of mammary gland development: the mammary end bud as a motile organ. Breast Cancer Res 2005, 7:245-251.

7. Howard BA, Gusterson BA: Human breast development. J Mammary Gland Biol Neoplasia 2000, 5:119-137.

8. Curtis Hewitt S, Couse JF, Korach KS: Estrogen receptor transcription and transactivation: estrogen receptor knockout mice: what their phenotypes reveal about mechanisms of estrogen action. Breast Cancer Res 2000, 2:345-352.

9. Bocchinfuso WP, Lindzey JK, Hewitt SC, Clark JA, Myers PH, Cooper R, Korach KS: Induction of mammary gland development in estrogen receptor-alpha knockout mice. Endocrinology 2000, 141:2982-2994.

10. Sakakura T, Nishizuka Y, Dawe CJ: Mesenchyme-dependent morphogenesis and epithelium-specific cytodifferentiation in mouse mammary gland. Science 1976, 194:1439-1441.

11. Cunha GR, Young P, Christov K, Guzman R, Nandi S, Talamantes $\mathrm{F}$, Thordarson G: Mammary phenotypic expression induced in epidermal cells by embryonic mammary mesenchyme. Acta Anat (Basel) 1995, 152:195-204.

12. Naylor MJ, Ormandy CJ: Mouse strain-specific patterns of mammary epithelial ductal side branching are elicited by stromal factors. Dev Dyn 2002, 225:100-105.

13. Daniel CW, Silberstein GB, Strickland P: Direct action of $\mathbf{1 7}$ beta-estradiol on mouse mammary ducts analyzed by sustained release implants and steroid autoradiography. Cancer Res 1987, 47:6052-6057.

14. Kleinberg DL, Feldman M, Ruan W: IGF-I: an essential factor in terminal end bud formation and ductal morphogenesis. J Mammary Gland Biol Neoplasia 2000, 5:7-17.

15. Gallego MI, Binart N, Robinson GW, Okagaki R, Coschigano KT, Perry J, Kopchick JJ, Oka T, Kelly PA, Hennighausen L: Prolactin, growth hormone, and epidermal growth factor activate Stat5 in different compartments of mammary tissue and exert different and overlapping developmental effects. Dev Biol 2001, 229:163-175.

16. Fisher $\mathrm{CR}$, Graves $\mathrm{KH}$, Parlow AF, Simpson ER: Characterization of mice deficient in aromatase (ArKO) because of targeted disruption of the cyp19 gene. Proc Natl Acad Sci USA 1998, 95:6965-6970.

17. Richards RG, Klotz DM, Walker MP, Diaugustine RP: Mammary gland branching morphogenesis is diminished in mice with a deficiency of insulin-like growth factor-I (IGF-I), but not in mice with a liver-specific deletion of IGF-I. Endocrinology 2004, 145:3106-3110.

18. Bonnette SG, Hadsell DL: Targeted disruption of the IGF-I receptor gene decreases cellular proliferation in mammary terminal end buds. Endocrinology 2001, 142:4937-4945.

19. Mueller SO, Clark JA, Myers PH, Korach KS: Mammary gland development in adult mice requires epithelial and stromal estrogen receptor alpha. Endocrinology 2002, 143:23572365.

20. Ruan W, Monaco ME, Kleinberg DL: Progesterone stimulates mammary gland ductal morphogenesis by synergizing with and enhancing insulin-like growth factor-I action. Endocrinology 2005, 146:1170-1178.

21. Soyal S, Ismail PM, Li J, Mulac-Jericevic B, Conneely OM, Lydon JP: Progesterone's role in mammary gland development and tumorigenesis as disclosed by experimental mouse genetics. Breast Cancer Res 2002, 4:191-196.

22. Brisken C, Park S, Vass T, Lydon JP, O'Malley BW, Weinberg RA: A paracrine role for the epithelial progesterone receptor in mammary gland development. Proc Natl Acad Sci USA 1998, 95:5076-5081.

23. Humphreys RC, Lydon J, O'Malley BW, Rosen JM: Mammary gland development is mediated by both stromal and epithelial progesterone receptors. Mol Endocrinol 1997, 11:801-811.

24. Brisken C, Heineman A, Chavarria T, Elenbaas B, Tan J, Dey SK, McMahon JA, McMahon AP, Weinberg RA: Essential function of Wnt-4 in mammary gland development downstream of progesterone signaling. Genes Dev 2000, 14:650-654.

25. Conneely OM, Jericevic BM, Lydon JP: Progesterone receptors in mammary gland development and tumorigenesis. J Mammary Gland Biol Neoplasia 2003, 8:205-214.

26. Fata JE, Kong YY, Li J, Sasaki T, Irie-Sasaki J, Moorehead RA, Elliott R, Scully S, Voura EB, Lacey DL, et al.: The osteoclast differentiation factor osteoprotegerin-ligand is essential for mammary gland development. Cell 2000, 103:41-50.

27. Coleman S, Silberstein GB, Daniel CW: Ductal morphogenesis in the mouse mammary gland: evidence supporting a role for epidermal growth factor. Dev Biol 1988, 127:304-315.

28. Kenney NJ, Bowman A, Korach KS, Barrett JC, Salomon DS: Effect of exogenous epidermal-like growth factors on mammary gland development and differentiation in the estrogen receptor-alpha knockout (ERKO) mouse. Breast Cancer Res Treat 2003, 79:161-173.

29. Sebastian J, Richards RG, Walker MP, Wiesen JF, Werb Z Derynck R, Hom YK, Cunha GR, DiAugustine RP: Activation and function of the epidermal growth factor receptor and erbB-2 during mammary gland morphogenesis. Cell Growth Differ 1998, 9:777-785.

30. Luetteke NC, Qiu TH, Fenton SE, Troyer KL, Riedel RF, Chang A Lee DC: Targeted inactivation of the EGF and amphiregulin genes reveals distinct roles for EGF receptor ligands in mouse mammary gland development. Development 1999 , 126:2739-2750.

31. Sternlicht MD, Sunnarborg SW, Kouros-Mehr H, Yu Y, Lee DC, Werb Z: Mammary ductal morphogenesis requires paracrine activation of stromal EGFR via ADAM17-dependent shedding of epithelial amphiregulin. Development 2005, 132:3923-3933.

32. Wiseman BS, Sternlicht MD, Lund LR, Alexander CM, Mott J, Bissell MJ, Soloway P, Itohara S, Werb Z: Site-specific inductive and inhibitory activities of MMP-2 and MMP-3 orchestrate mammary gland branching morphogenesis. J Cell Biol 2003, 162:1123-1133.

33. Kheradmand F, Rishi K, Werb Z: Signaling through the EGF receptor controls lung morphogenesis in part by regulating MT1-MMP-mediated activation of gelatinase A/MMP2. J Cell Sci 2002, 115:839-848.

34. Lu P, Ewald A, Martin G, Werb Z: Essential function of FGF signaling pathway during branching morphogenesis of mammary gland (abstract \#515). Dev Biol 2005, 283:680-681.

35. Jackson-Fisher AJ, Bellinger G, Ramabhadran R, Morris JK, Lee $\mathrm{KF}$, Stern DF: ErbB2 is required for ductal morphogenesis of the mammary gland. Proc Natl Acad Sci USA 2004, 101: 17138-17143.

36. Andrechek ER, White D, Muller WJ: Targeted disruption of ErbB2/Neu in the mammary epithelium results in impaired ductal outgrowth. Oncogene 2005, 24:932-937. 
37. Tidcombe $\mathrm{H}$, Jackson-Fisher A, Mathers $\mathrm{K}$, Stern DF, Gassmann $M$, Golding JP: Neural and mammary gland defects in ErbB4 knockout mice genetically rescued from embryonic lethality. Proc Natl Acad Sci USA 2003, 100:8281-8286.

38. Dunbar ME, Dann P, Brown CW, Van Houton J, Dreyer B, Philbrick WP, Wysolmerski JJ: Temporally regulated overexpression of parathyroid hormone-related protein in the mammary gland reveals distinct fetal and pubertal phenotypes. J Endocrino/ 2001, 171:403-416.

39. Srinivasan K, Strickland P, Valdes A, Shin GC, Hinck L: Netrin-1/ neogenin interaction stabilizes multipotent progenitor cap cells during mammary gland morphogenesis. Dev Cell 2003, 4:371-382.

40. Lewis MT: Hedgehog signaling in mouse mammary gland development and neoplasia. J Mammary Gland Biol Neoplasia 2001, 6:53-66.

41. Radisky DC, Hirai Y, Bissell MJ: Delivering the message: epimorphin and mammary epithelial morphogenesis. Trends Cell Biol 2003, 13:426-434.

42. Gouon-Evans V, Lin EY, Pollard JW: Requirement of macrophages and eosinophils and their cytokines/chemokines for mammary gland development. Breast Cancer Res 2002, 4:155-164.

43. Daniel CW, Robinson S, Silberstein GB: The role of TGF- $\beta$ in patterning and growth of the mammary ductal tree. J Mammary Gland Biol Neoplasia 1996, 1:331-341.

44. Ewan KB, Shyamala G, Ravani SA, Tang Y, Akhurst R, Wakefield $\mathrm{L}$, Barcellos-Hoff $\mathrm{MH}$ : Latent transforming growth factor-beta activation in mammary gland: regulation by ovarian hormones affects ductal and alveolar proliferation. Am J Pathol 2002, 160:2081-2093.

45. Sternlicht MD, Werb Z: How matrix metalloproteinases regulate cell behavior. Annu Rev Cell Dev Bio/ 2001, 17:463-516.

46. Fata JE, Werb Z, Bissell MJ: Regulation of mammary gland branching morphogenesis by the extracellular matrix and its remodeling enzymes. Breast Cancer Res 2004, 6:1-11.

47. Chen J, Diacovo TG, Grenache DG, Santoro SA, Zutter MM: The $\alpha_{2}$ integrin subunit-deficient mouse: a multifaceted phenotype including defects of branching morphogenesis and hemostasis. Am J Pathol 2002, 161:337-344.

48. Klinowska TC, Soriano JV, Edwards GM, Oliver JM, Valentijn AJ, Montesano R, Streuli $\mathrm{CH}$ : Laminin and $\beta_{1}$ integrins are crucial for normal mammary gland development in the mouse. Dev Biol 1999, 215:13-32.

49. Klinowska TC, Alexander CM, Georges-Labouesse E, Van der Neut R, Kreidberg JA, Jones CJ, Sonnenberg A, Streuli CH: Epithelial development and differentiation in the mammary gland is not dependent on $\alpha_{3}$ or $\alpha_{6}$ integrin subunits. Dev Biol 2001, 233:449-467.

50. Vogel WF, Aszodi A, Alves F, Pawson T: Discoidin domain receptor 1 tyrosine kinase has an essential role in mammary gland development. Mol Cell Biol 2001, 21:2906-2917.

51. Hathaway HJ, Shur BD: Mammary gland morphogenesis is inhibited in transgenic mice that overexpress cell surface $\beta 1,4-$ galactosyltransferase. Development 1996, 122:28592872.

52. Muschler J, Levy D, Boudreau R, Henry M, Campbell K, Bissell MJ: A role for dystroglycan in epithelial polarization: loss of function in breast tumor cells. Cancer Res 2002, 62:7102-7109.

53. Sakai $T$, Larsen $M$, Yamada KM: Fibronectin requirement in branching morphogenesis. Nature 2003, 423:876-881.

54. Gudjonsson T, Ronnov-Jessen L, Villadsen R, Rank F, Bissell MJ, Petersen OW: Normal and tumor-derived myoepithelial cells differ in their ability to interact with luminal breast epithelial cells for polarity and basement membrane deposition. $J$ Cell Sci 2002, 115:39-50.

55. Runswick SK, O'Hare MJ, Jones L, Streuli CH, Garrod DR: Desmosomal adhesion regulates epithelial morphogenesis and cell positioning. Nat Cell Biol 2001, 3:823-830. 